\title{
Laboreal
}

Volume 15 N$^{\circ} 2$ | 2019

Varia

\section{Trabajo, subjetividad y clínicas del trabajo : una aproximación al estado de su cuestión en el caso colombiano}

Trabalho, subjetividade e clinicas do trabalho : uma aproximação ao estado da questão no caso colombiano

Travail, subjectivité et clinique du travail : approximation de l'etat de question dans l'affaire colombien

Work, subjectivity and clinics of work : an approximation to the state of the art in the colombian case

Johnny Orejuela, María del Mar Pérez y Andrés Vásquez

\section{OpenEdition}

\section{Journals}

Edición electrónica

URL: http://journals.openedition.org/laboreal/15522

DOI: $10.4000 /$ laboreal. 15522

ISSN: 1646-5237

Editor

Universidade do Porto

Referencia electrónica

Johnny Orejuela, María del Mar Pérez y Andrés Vásquez, «Trabajo, subjetividad y clínicas del trabajo una aproximación al estado de su cuestión en el caso colombiano », Laboreal [En línea], Volume 15 No2 | 2019, Publicado el 01 diciembre 2019, consultado el 11 abril 2020. URL : http:// journals.openedition.org/laboreal/15522 ; DOI : https://doi.org/10.4000/laboreal.15522

Este documento fue generado automáticamente el 11 abril 2020.

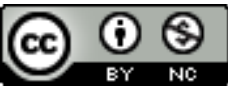

Laboreal está licenciado com uma Licença Creative Commons - Atribuição-NãoComercial 4.0 Internacional. 


\section{Trabajo, subjetividad y clínicas del trabajo : una aproximación al estado de su cuestión en el caso colombiano}

Trabalho, subjetividade e clinicas do trabalho : uma aproximação ao estado da questão no caso colombiano

Travail, subjectivité et clinique du travail : approximation de l'etat de question dans l'affaire colombien

Work, subjectivity and clinics of work : an approximation to the state of the art in the colombian case

Johnny Orejuela, María del Mar Pérez y Andrés Vásquez

\section{NOTA DEL EDITOR}

Manuscrito recibido en septiembre/2019

Aceptado tras peritaje noviembre/2019

\section{Presentación}

1 Este trabajo presenta el balance sobre la investigación alrededor de los desarrollos de las clínicas del trabajo, en general, y de la psicodinámica del trabajo, en particular, en el contexto académico colombiano. La relación trabajo-subjetividad comienza a ser abordada como foco central de las clínicas del trabajo, y más específicamente desde la psicodinámica del trabajo, desarrollada por su pionero Christophe Dejours y ampliada particularmente en Brasil por los trabajos de Laerte Idal Sznelwar, Selma Lancman, Seiji Ushida, Juliana de Oliveira Barros, en la Universidad de São Paulo; Ana Magnolia 
Mendes, desde la Universidad de Brasilia y Álvaro Merlo, en la Universidad de Rio Grande do Sul.

En Colombia, la aparición de trabajos desde esta perspectiva es reciente, debido en parte a la poca difusión en español de esta perspectiva de análisis. No obstante, se encuentran algunos estudios que han abordado el problema de la relación trabajosubjetividad, que, aunque no se suscriben como clínicas del trabajo propiamente, sí señalan interés por descifrar los efectos del trabajo sobre la subjetividad y de la participación de ésta en el desarrollo de la actividad laboral.

\section{Las clínicas del trabajo : una perspectiva clínico- crítica para el análisis/intervención de la relación trabajo-subjetividad-contexto}

En Francia, hacia 1980, emergió el subcampo de la psicodinámica del trabajo dentro del amplio y tradicional campo de la psicología de las organizaciones y del trabajo. Apareció en cabeza del psicopatólogo del trabajo y también psicoanalista Christophe Dejours y su propósito inicial fue estudiar las psicopatologías derivadas del mundo laboral, para luego abarcar el lugar del trabajo en la economía psíquica de los sujetos y la significatividad anímica del trabajo como organizador socioemocional.

La novedad de esta perspectiva fue que superó los prejuicios usando la perspectiva clínica para analizar el trabajo y sus efectos sobre la subjetividad en clave de experiencias de malestar subjetivo, sufrimiento y placer. Igualmente, inspiró a leer este objeto (la relación trabajo-subjetividad) a partir de otros marcos teóricos referenciales como los de la teoría de la actividad de Vygotsky y las sociología clínico-crítica y ergonomía francesas. Todo esto dio lugar al surgimiento de tres perspectivas clínicas del trabajo: la psicodinámica del trabajo, la clínica de la actividad y la sociología clínica (Tabla 1). De aquí que no se trata de una clínica del trabajo en singular, (no es una sola), sino de las clínicas del trabajo, en plural (Lhuilier, 2006 ; Orejuela, 2018 ; Mendes, Araujo, \& Duarte, 2014).

Tabla 1 : Las clínicas del trabajo : un campo, tres perspectivas

\begin{tabular}{|c|c|c|}
\hline LA PSICODINÁMICA DEL TRABAJO & LA CLÍNICA DE LA ACTIVIDAD & LA SOCIOLOGÍA CLINIICA \\
\hline $\begin{array}{l}\text { Aproximación que emerge a partir de la } \\
\text { "re descripción" de la psicopatologia del } \\
\text { trabajo de origen francesa, por una lectura } \\
\text { muy particular del psicoanálisis (Lapanche) } \\
\text { hecha por: }\end{array}$ & $\begin{array}{l}\text { Abordaje que se enfoca en el análisis del } \\
\text { trabajo valiéndose de dos instrumentos: la } \\
\text { psicologia rusa de Vygotsky y la psicologia } \\
\text { ergonómica francesa, teniendo como } \\
\text { principal exponente a: }\end{array}$ & $\begin{array}{l}\text { Perspectiva en las que integradas el } \\
\text { psicoanálisis con la sociologia critica } \\
\text { se apuesta por una comprensión de los } \\
\text { origenes sociológicos del sufrimiento, } \\
\text { del sufrimiento en el campo de lo social } \\
\text { (violencia, exclusión, humillación, } \\
\text { resentimiento). Sus exponentes son: }\end{array}$ \\
\hline $\begin{array}{l}\text { Cristophe Dejours, ampliada en Brasil por } \\
\text { Laerte Idal Sznelwar, Selma Lancman, Seiji } \\
\text { Ushida, Juliana de Oliveira Barros, Ana } \\
\text { Magnolia Mendes y Álvaro Merlo. }\end{array}$ & Yves Clot. & Vincent de Gaulejac y Eugene Enriquéz. \\
\hline
\end{tabular}

5 Las clínicas del trabajo hacen parte de la psicología organizacional y del trabajo, pero no se reducen a ella, sino que pueden ser entendidas como una propuesta sub- 
disciplinar, con identidad propia (al interior de la psicología aplicada a las organizaciones y el trabajo), y, a la vez, transdisciplinar (recoge y re-articula conceptos de la antropología, la sociología, la filosofía, el psicoanálisis, la medicina, etc.). Así mismo, el campo de clínicas del trabajo, particularmente la psicodinámica del trabajo, propenden a ayudar a superar tres supuestos hiatos : el primero, la incompatibilidad entre el mundo subjetivo y el mundo del trabajo ; segundo, el aparente abismo entre la Psicología Clínica y la Psicología Organizacional y del Trabajo; y tercero, el supuesto infundado de una incompatibilidad entre el Psicoanálisis y el estudio de las Organizaciones y del Trabajo.

6 La perspectiva de las clínicas del trabajo ha mostrado valor heurístico y fecundidad teórica, investigativa y práctica al recoger ampliamente estudios sobre temas que constituyen los conceptos de la psicodinámica del trabajo, alrededor de los cuales gravitan teoría e investigación, a la vez que sirven de marco teórico de inteligibilidad de la subjetividad implicada en la actividad laboral : placer en el trabajo, sufrimiento laboral (patogénico, creativo, ético), estrategias de defensa e inteligencia desplegada, movilización subjetiva, trabajo real, trabajo prescrito, real de trabajo, defensa colectiva y colectivo de trabajo, psicodinámica del reconocimiento (juicio de utilidad y juicio de belleza), etc. Toda una gramática conceptual claramente diferenciada de los intereses de la psicología organizacional tradicional, de origen norteamericano y cuño funcionalista, que se ha centrado en el análisis del trabajo organizado, formal, consistente con la organización clásica del trabajo (Taylor-fordista) y la teoría del capital humano de T. Shultz, y cuyos ejes temáticos han sido: la cultura, la selección, el clima, el cambio, la estructura, el liderazgo, el conflicto, los grupos y equipos, el compromiso y los valores organizacionales, entre otros.

7 Los pioneros y autores representativos de la psicodinámica del trabajo son C. Dejours, D. Lhuilier, P. Molinier (por citar solo algunos de Francia) y S. Uchida, L. Sznelwar, J. O. Barros, S. Lancman (2011), Ana Magnolia Mendes y Álvaro Merlo (para el caso de Brasil). En el área francófona de Canadá esta subdisciplina también ha tenido amplio desarrollo. Sin embargo, hemos de advertir que esta tendencia es poco desarrollada en América Latina, y prácticamente desconocida entre una gran mayoría de investigadores hispanos y, obviamente, colombianos, lo cual explica que sea una perspectiva de análisis e investigación, si no inédita, sí poco desplegada y difundida en nuestro país.

8 Las clínicas del trabajo, y más enfáticamente la perspectiva psicodinámica del trabajo como una de ellas, son una perspectiva del análisis de la relación trabajo-subjetividad, en la que se reconocen tres aspectos importantes: (a) la dimensión singular del sujeto trabajador ; (b) la centralidad del trabajo en la economía psíquica (psicodinámica) y (c) la organización como contexto especifico de relaciones intersubjetivas y marco simbólico que pauta el orden de la interacción en las mismas (Tabla 2). Es un abordaje que implica el uso del método clínico, que quizás a los psicólogos organizacionales les parezca inusual y hasta extraño que pueda articularse al análisis de problemáticas laborales (labor-reales : laborales reales, o de lo real presente en lo laboral que siempre demanda la recursividad subjetiva para superarlo - como bien lo sugiere el nombre mismo de esta revista), porque tienden a asociarlo de manera inmediata y acrítica con la psicoterapia, lo cual no es así necesariamente.

9 Además, la psicodinámica del trabajo, como una de las clínicas del trabajo, por lo menos en la opinión de Mendes et al. (2014), podría decirse que constituye a la vez una propuesta de intervención clínico-terapéutica-ampliada en el contexto de trabajo, lo 
cual supera el prejuicio de que es desaconsejable, e incluso poco ético, procurar tener efectos psicoterapéuticos en el contexto laboral. Las experiencias de intervención clínica con trabajadores de Telecom en Francia (Dejours \& Bègue, 2010) y de sindicatos de docentes y personal bancario en Brasil (Mendes et al., 2014), derrumban este prejuicio, que solo es una racionalización ideológica en la psicología, que carece de fundamento y más bien evidencia lo poco que se conoce este campo subdisciplinar en nuestro país.

En términos generales, se podría decir que las clínicas del trabajo, y en particular la psicodinámica del trabajo, tienen por objeto de estudio la relación trabajo-subjetividadcontexto ; y, por método, el clínico.

Las clínicas del trabajo no tratan de una clínica del diván enfocada en problemáticas singulares girando en torno a las fantasmáticas individuales. Tampoco se trata de un abordaje que diluye al sujeto en estructuras sociales que lo anteceden. El énfasis de la palabra clínica en ese caso es sobre la articulación del mundo psíquico con el mundo social... Y no siendo una clínica exclusivamente del sujeto intrapsíquico, pero sin desconocerlo, ella incorpora en sus cuestionamientos "la producción social del sufrimiento en el trabajo" (Bendassolli \& Soboll, 2011, p. 3, traducción libre).

Tabla 2. Triple dimensión del análisis de la relación sujeto-trabajo en las clínicas del trabajo

\begin{tabular}{|c|c|c|}
\hline EL SUJETO & EL TRABAJO & $\begin{array}{l}\text { LA ORGANIZACIÓN/ } \\
\text { MERCADO DE TRABAJO/ SOCIEDAD }\end{array}$ \\
\hline $\begin{array}{l}\text { El agente } \\
\text { (con capacidad de acción y reflexividad) }\end{array}$ & $\begin{array}{l}\text { La actividad } \\
\text { (de carácter económico-productivo, } \\
\text { cargada de sentido socio históricamente } \\
\text { situada) }\end{array}$ & $\begin{array}{l}\text { El contexto } \\
\text { (estructura simbólica que pauta el orden de } \\
\text { relaciones y sentido) }\end{array}$ \\
\hline
\end{tabular}

11 Así, en nuestra consideración, las clínicas del trabajo pueden entenderse como una apuesta crítica de carácter teórico-clínico-terapéutico-investigativo que se propone ayudar a comprender, visibilizar y superar el malestar y sufrimiento propios del mundo del trabajo. Con esto, se podría decir en sentido amplio, que, las clínicas del trabajo, y particularmente la psicodinámica del trabajo, son una apuesta etho-política comprometida con tres aspectos fundamentales: (a) denunciar el sufrimiento invisibilizado que implica el trabajo bajo la gramática de la fragmentación precarizante que impone el nuevo espíritu del capitalismo, (b) la no banalización de la injusticia social presente en el mundo del trabajo y (c) aportar un dispositivo de "cura" (dispositivo de escucha colectiva) para el sufrimiento experimentado en el trabajo.

\section{La relación trabajo-subjetividad como foco de investigación en Colombia}

\subsection{La tradición en psicología del trabajo y de las organizaciones}

12 En las principales universidades públicas y privadas de Colombia existe una fuerte tradición de formación en psicología del trabajo y de las organizaciones. Está presente 
en casi todos los programas de formación; es la segunda área de aplicación de la psicología después de la psicóloga clínica y la primera con mayor índice de empleabilidad y calidad de empleo entre los psicólogos profesionales (Orejuela, Bermúdez, Urrea, \& Delgado, 2013). Esto ha permitido la proliferación de programas de formación de pregrado y posgrado en psicología de las organizaciones, del trabajo y de los recursos humanos (campo también denominado gestión humana), lo que a su vez ha derivado en publicaciones nacionales e internacionales, pero de manera dispersa.

En Colombia no existe una revista especializada en este campo pese al alto volumen de producción científica en él, lo que señala la necesidad de un órgano difusor especializado y de alto impacto que concentre la producción nacional a este respecto, porque existe un conjunto de investigaciones y publicaciones derivadas de los trabajos de grado e investigaciones desarrolladas en los principales grupos de investigación de los diferentes departamentos y facultades de psicología, que toman como foco la relación trabajo-subjetividad.

La subjetividad puede expresarse en diferentes dimensiones, según la relación con el trabajo: La subjetividad laboral, la identidad laboral, el contrato psicológico, los sentidos atribuidos al trabajo, la valoración subjetiva del trabajo, y, por supuesto, las experiencias de placer, malestar y sufrimiento en el trabajo, que han sido objeto de investigación en el contexto colombiano. Cabe destacar el papel de los grupos de investigación en el proceso de formación de investigadores y sobre el abordaje de estos temas. Los propósitos de los grupos se centran de manera específica en la psicología del trabajo y las organizaciones, o en líneas de trabajo, aunque adscritas a grupos mayores y generalistas, reconocidos por el sistema de ciencia y tecnología colombiano (Colciencias). Estos grupos se adscriben a universidades a redes, así como del Nodo en Psicología Organizacional y del Trabajo (POT), adscrito a la Red Colombiana de Investigadores en Psicología, auspiciada por la Asociación Colombiana de Facultades de Psicología (Ascofapsi).

15 Es de destacar el papel que ha cumplido el nodo de investigación en POT en la articulación de los investigadores especializados en este campo y como un espacio de difusión de la investigación en general, así como también, más recientemente, la investigación asociada a las clínicas del trabajo. El nodo ha capitalizado los esfuerzos de investigación en los que la temática trabajo-subjetividad ha sido abordada hasta el momento en tres proyectos editoriales, que han consolidado la investigación nacional, con algunas participaciones internacionales, entre las que se encuentran la de la profesora Ana Magnolia Mendes como una de las principales representantes de la clínica del trabajo en América Latina.

El primero de los tres volúmenes titulado Psicología Organizacional y del Trabajo: Reflexiones y experiencias de investigación (Rentería \& Aguilar, 2009), aborda temas como : la construcción de sujetos en las nuevas realidades laborales (Peralta, 2009); el contrato psicológico y las nuevas realidades del trabajo (Vesga, 2009); una lectura crítica de las operaciones de la Psicología en su relación con el trabajo (Pulido, 2009); la fatiga laboral como una consecuencia en la salud derivada de la organización del trabajo (Fang, 2009); el Mobbing y la violencia en las organizaciones (Giraldo, Aguilar \& González, 2009), y la promoción de la salud en el lugar de trabajo (Gómez, 2009), entre otros.

17 El segundo volumen, Psicología de las Organizaciones y del trabajo: Apuestas de investigación (Orejuela, 2014a), discute en varios de sus capítulos sobre la relación trabajo- 
subjetividad, aludiendo a temas tales como: el significado del trabajo (Del Carpio, Álvaro \& Garrido, 2014); la identidad profesional y la agencia (Andrade, 2014). El contrato psicológico (Vesga, 2014); la motivación y satisfacción laboral (García \& Forero, 2014); la fatiga laboral (Neisa, Godoy \& Tangarife, 2014) las estrategias de afrontamiento del estrés (Montoya \& Panesso, 2014), entre otros. Además, en este volumen se aborda por primera vez y de manera explícita la temática de las clínicas del trabajo, mediante un estado de la cuestión en América Latina (Melo \& Orejuela, 2014), un trabajo sobre la valoración subjetiva del trabajo en clave de placer y sufrimiento (Orejuela, 2014c); y la escucha clínica y política del sufrimiento en el trabajo presentada por Ana Magnolia Mendes (Mendes et al., 2014), entre otros, que se describirán más adelante.

El tercer volumen, Psicología de las Organizaciones y del trabajo : Apuestas de investigación II (Orejuela, Andrade \& Villamizar, 2014), incluye trabajos que aluden a la relación trabajo-subjetividad como: el acoso laboral (Araujo, 2014); las actitudes frente a la reintegración laboral (Grueso, Antón \& Lopez-Santamaia, 2016); el contagio emocional en las organizaciones (Delgado, 2016); el estrés y su afrontamiento en el ámbito organizacional (García \& Vélez 2016), entre otros. También son abordados de nuevo temas específicos en clínica del trabajo como: el malestar en el trabajo, el método clínico y el trabajo con grupos en las organizaciones, la experiencia clínica con un grupo de enfermeras en su lugar de trabajo y el trabajo como un factor productor de sufrimiento subjetivo, entre otros que también se describirán en detalle.

Aparte del nodo de investigación en POT, también se deben resaltar en este mapa académico del abordaje de la relación trabajo-subjetividad los desarrollos del grupo de investigación en Psicología Organizacional y del Trabajo, dirigido por el profesor Érico Rentería, y del doctorado en psicología de la Universidad del Valle, en el cual, cabe subrayar, se han formado la mayoría de los doctores en psicología del trabajo en el país, con trabajos que involucran : "Contenido y extensión del contrato psicológico según la modalidad de trabajo" (Vesga, 2015) ; "Carrera, identidad y trayectorias en condiciones de trabajo fragmentado" (Giraldo, 2015); "El burnout como una metáfora para comprender las relaciones y contextos de trabajo desde la salud" (Díaz, 2017); "El significado del trabajo y el sentido de la producción académica" (Romero, 2017); "La configuración de la identidad profesional en trabajadores de alta empleabilidad" (Andrade, 2015) ; y, "Emprendimiento, gubernamentalidad y subjetividad" desarrollada por Deidi Maca (2019), bajo la orientación del profesor Nelson Molina

En trabajos de pregrado y maestría se destacan algunos que han derivado en publicaciones nacionales e internacionales. : "La identidad profesional de dirigentes de organizaciones no gubernamentales" (Torres, 2010); "La identidad profesional y el desarrollo de carrera en mujeres gerentes" (Galves \& Garcés, 2013) ; y, "La construcción de la identidad de profesionales en oficiales de policía” (García, 2014).

21 Finalmente, otro polo de desarrollo de la investigación sobre la relación trabajosubjetividad ha estado en el grupo de investigación Estudios Críticos de las Organizaciones $y$ del Trabajo de la Universidad Javeriana, bajo la dirección del profesor Hernán Camilo Pulido $(2012,2013,2018)$, en el cual, desde la perspectiva de una psicología crítica, se han abordado temas relacionados con el trabajo y la subjetividad en clave de identidad, la subjetividad laboral y patologización y despatologización de los discursos psicológicos (Pulido \& Carvajal, 2014), entre otros. 


\subsection{El desarrollo de la investigación sobre la relación trabajo- subjetividad} En cuanto a concentración, las temáticas de la subjetividad laboral (Orejuela \& Ramírez, 2011) y los sentidos del trabajo (Corredor, Páramo, \& Orejuela, 2018 ; Romero, Blanch, \& Rentería, 2016) son quizás las líneas que mayor número de trabajos investigativos y publicaciones sobre expresiones de la relación trabajo-subjetividad. En cuanto a la difusión de este conocimiento especializado se resalta el papel que han jugado revistas como Universitas Pshychologica, (Universidad Javeriana, Bogotá), Diversitas, (Universidad Santo Tomás), Psicología desde el Caribe, (Universidad del Norte), Pensamiento Psicológico, (Universidad Javeriana, Cali), y Revista Guillermo de Ockham (Universidad de San Buenaventura, Cali) ; y, el Congreso Colombiano de Psicología, pues desde 2015 ha incluido la perspectiva clínica del trabajo mediante simposios y ponencias que presentan y discuten los temas relativos a la perspectiva Clínica del Trabajo y al placer y sufrimiento en el trabajo como manifestaciones de la subjetividad.

Finalmente, si bien las relaciones trabajo-subjetividad y salud mental o psíquica han tenido una amplia discusión, sobre todo desde la perspectiva de la psicología de la salud ocupacional y de los riesgos psicosociales (Lemos, Calle, Roldán, Valencia, Orejuela, \& Román-Calderón, 2019), incluso desde la perspectiva de la felicidad y el bienestar psicológico en el trabajo (Sanín, 2016), y de las organizaciones saludables (Grueso, 2016), aun en el país no existe un evento de envergadura que aglutine a los investigadores para discutir las implicaciones del trabajo sobre la subjetividad desde una perspectiva Clínica del Trabajo, debido precisamente a que es una perspectiva aun poco conocida en nuestro contexto y aun no logra consolidar la masa crítica suficiente. Como en el caso de las revistas, estas participaciones están dispersas en eventos más amplios de psicología general o de salud ocupacional. Hay mora de plantear un evento desde la psicología que permita socializar los diferentes esfuerzos de investigación y formación alrededor de la relación trabajo-subjetividad en una perspectiva clínica, ya que quizás ahora exista un volumen de trabajos suficientes que puedan dar lugar a un evento de tal naturaleza. 


\section{La perspectiva de las clínicas del trabajo y las organizaciones en Colombia}

\subsection{Presencia de las clínicas del trabajo en la formación de pregrado}

Si bien la producción en español sobre la relación trabajo-subjetividad en una perspectiva clínica del trabajo aún es limitada, desde 2014 se ha iniciado con fuerza en Colombia la difusión de esta perspectiva en varias vías : trabajos de grado de pregrado y posgrado, publicaciones, participación en congresos y formaciones específicas en el tema.

En cuanto a las participaciones en congresos, en 2015 el autor principal de este texto presentó por primera vez en la historia académica del campo de la Psicología Organizacional y del Trabajo una conferencia central sobre Clínicas del trabajo, titulada "Las clínicas del trabajo: una perspectiva de análisis de la relación sujeto-trabajo en tiempos de fragmentación laboral". Esta presentación ocurrió en el Congreso Colombiano de Psicología, evento organizado por la Asociación Colombiana de Facultades de Psicología (Ascofapsi) y el Colegio Colombiano de Psicología (Colpsic). En dicho congreso también se presentó el primer taller pre-congreso sobre esta temática titulado : "El malestar en el trabajo : elementos para un diagnóstico crítico en tiempo de fragmentación del trabajo".

Para 2017, esta perspectiva volvió a presentarse en el Congreso Colombiano de Psicología, como simposio entre profesores de la Universidad de Antioquia y la Universidad EAFIT titulado : "Clínica Analítica de las organizaciones y del trabajo". En este mismo congreso se presentó también la conferencia central : “Teoría y práctica de la mediación, una lectura desde la perspectiva de Gilbert Simondon y el Método analítico en las organizaciones", por parte del doctor en Administración Carlos Mario Henao, de la Universidad EAFIT.

En noviembre de 2016, en la Institución Universitaria Cesmag, de la ciudad de Pasto, se presentó la conferencia central "El malestar laboral en tiempos de fragmentación", en el marco del VII Congreso Internacional Perspectivas y Abordajes de la Psicología Clínica en la Actualidad. Cabe indicar también que, previo a la explosión de trabajos sobre esta subdisciplina después de 2014, el profesor Horacio Manrique había hecho una avanzada en la presentación de una comprensión desde el método clínico del mundo de las organizaciones y del trabajo en el III Congreso Nacional de Psicología Colpsic-Ascofapsi del año 2013, con la ponencia "Clínica de las organizaciones : una aplicación del método analítico"; y en el XVIII Congreso Internacional de Psicoterapia de Grupo y Procesos Grupales, en julio de 2012 en Cartagena, participó con la ponencia "Clínica de las organizaciones", una perspectiva aún más inusual en nuestro contexto académico para esa fecha.

Recientemente, en octubre de 2018 hubo participación de diez trabajos de autores colombianos en el Simposio franco-latinoamericano sobre subjetividad y trabajo: entre el malestar y el bien estar: entre ellos se destacan los siguientes trabajos por ser específicamente desarrollados en la perspectiva clínica psicodinámica del trabajo : la negación del reconocimiento como determinante del malestar en el trabajo: unas lectura entre Honneth y Dejours, ponencia central presentada por Johnny Orejuela; La evaluación del 
desempeño : entre la sujeción del trabajador y lo humano e inhumano en la organización por Sergio René Oquendo Puerta y Héctor L. Bermúdez de la Universidad de Antioquia; Experiencias de sufrimiento laboral en trabajadores por Iván David Jojoa Arcos, Canady Ordoñez Pérez y Juan Pablo Mora de la Institución Universitaria Cesmag; Sentidos atribuidos a las experiencias de reconocimiento por un grupo de investigadores de una universidad pública colombiana por Daniel Ernesto Gómez de la Maestría en Psicología de la Universidad de Antioquia; Escenarios de expresión oral para tramitar el sufrimiento laboral por Iván David Jojoa Arcos y Johana Trujillo Zambrano de la Institución Universitaria Cesmag de Pasto, Nariño.

De igual manera, en la Facultad de Psicología de la Universidad de San Buenaventura, en Cali, la profesora María del Pilar Murcia ha dirigido también tres Trabajos de Grado a nivel de pregrado en el campo de las clínicas del trabajo, los cuales han versado sobre la dimensión dialéctica del trabajo como fuente de placer y sufrimiento (Giraldo, 2018); los efectos de la negación del reconocimiento en la autopercepción de docentes (Londoño, 2015); y las experiencias de placer y sufrimiento en trabajadoras sexuales (López, 2017).

31 En paralelo a la divulgación, en la formación se dio un primer ejercicio en el ámbito formal entre 2016 y 2017. Desde la Universidad EAFIT se ofreció el primer diplomado en el país que apuntó a presentar y formar desde una perspectiva clínica para el análisis de las organizaciones y del trabajo, y que llevó por nombre Diplomado en Clínica analítica de las organizaciones. De igual manera, el programa de Maestría en Psicología que próximamente ofrecerá la Universidad EAFIT tendrá una línea de énfasis en clínica de las organizaciones y del trabajo.

En esta misma línea de formación, los profesores Carlos Mario Henao y Horacio Manrique dirigieron en la Universidad EAFIT entre 2009 y 2014 el grupo de estudio Gestión humana de orientación analítica; del que surgió la idea de realizar el proyecto de investigación Clínica de las organizaciones: una aplicación del método analítico, bajo el auspicio del Grupo de investigación interinstitucional El método analítico y sus aplicaciones en las ciencias sociales y humanas (Universidad EAFIT y Universidad de Antioquia).

En relación con la formación se destacan también ya varios trabajos de grado de pregrado, especialización, maestría y doctorado, que se han desarrollado en la perspectiva de una clínica del trabajo y las organizaciones. En pregrado, la profesora Angélica Delgado de la Universidad de San Buenaventura dirigió el trabajo "La investigación como trabajo, ¿un escenario de placer, sufrimiento y lucha por el reconocimiento ? : un estudio en un grupo de ocho investigadores de la ciudad de Cali" (Quiroga \& Valencia, 2016). Por su parte, Angélica Meléndez (2018), bajo la supervisión del profesor Wilner Riascos, desarrolló el trabajo "Causas, síntomas y estrategias de defensa del malestar y el sufrimiento en altos mandos de la ciudad de Cali".

En el pregrado en Psicología de la Universidad EAFIT los estudiantes han comenzado a interesarse por las Clínicas del Trabajo y su abordaje de problemáticas relativas al placer y el sufrimiento y así han escogido diferentes campos de verificación empírica de esta perspectiva clínico-teórica. Eugenia Durando (2019) se interesó por comprender las "Vivencias de placer y sufrimiento en el trabajo como Chef" y su trabajo de grado fue reconocido como meritorio por su novedad y rigor ; Alejandro Correa (2019) se interesó en conocer las "Experiencias de malestar, sufrimiento y placer en el trabajo de pilotos de aerolíneas Charter" ; Andrés Felipe López (2019) indagó las experiencias de "Placer y sufrimiento en el proceso de sucesión de empresa familiares”; Anderson Gañan (2019) 
indagó para conocer las "Experiencias de placer y sufrimiento en el trabajo de periodistas"; Javier Cardona (2019) se preocupó por el "Placer y sufrimiento en trabajadores emprendedores" y, debido a las recurrentes quejas de malestar en la formación y realización de la tesis doctoral, Laura Aristizabal (2019) se interesó por realizar una investigación sobre las vivencias de placer y sufrimiento en este nivel de formación.

Por su parte, la profesora Adaneis Álvarez, en el programa de Psicología de la Universidad Católica de Pereira dirigió el trabajo "El sufrimiento del sujeto por el trabajo : una aproximación psicoanalítica" (García, 2015). En 2019, en el departamento de psicología de la Universidad EAFIT, se fundó el primer semillero de investigación sobre clínica del trabajo y las organizaciones, bajo el liderazgo del estudiante Anderson Gañan, y en concordancia con la línea de investigación del mismo nombre de la Maestría en Psicología del mismo departamento. De la misma manera, los profesores Sergio Oquendo y Héctor Bermúdez lideran el grupo de estudio adscrito a la maestría en gestión humana y el grupo de investigación Comportamiento Humano en la Organización (Comphor) de la Universidad de Antioquia, en el que se debaten temas sobre la relación trabajo-subjetividad en perspectiva Clínica del Trabajo.

Estas dos iniciativas, inéditas a la fecha en Colombia, indican el crecimiento del interés por la Clínica del Trabajo en nuestro país. Si bien hasta el momento no existe una maestría especializada en Clínica del Trabajo, es de reconocer que la Maestría en Psicología de la Universidad de EAFIT es la primera en Colombia en desplegar una línea de énfasis en Clínica del Trabajo y las Organizaciones, con lo que espera servir de semillero para la formación de investigadores con esta perspectiva, y que sus esfuerzos se traduzcan en una mayor investigación y publicación en el país.

Finalmente, otra de las universidades que ha comenzado a desarrollar trabajos de formación en investigación sobre la psicodinámica del trabajo ha sido la Universidad Cesmag de Pasto, Nariño, bajo la dirección del profesor Iván David Jojoa Arcos se han desarrollado los trabajos de pregrado: "Experiencias de sufrimiento laboral en trabajadores" (Ordoñez \& Mora, 2018) y "Escenarios de expresión oral para tramitar el sufrimiento laboral" (Trujillo, 2018).

\subsection{Presencia de las clínicas del trabajo en la formación de posgrado}

En el marco de la Especialización en Psicología Clínica con Orientación Psicoanalítica de la Universidad de San Buenaventura, Cali : Johnny Orejuela dirigió los trabajos: "La significatividad anímica del trabajo : una lectura psicoanalítica del valor del trabajo en la constitución del sujeto" (Ruiz, 2015) y "Pasando del malestar al sufrimiento : lo no dicho que se escucha a través del síntoma de un docente" (Murcia, 2015). Igualmente, Sandra Sanabria (2016) desarrolló una investigación alrededor de "El sufrimiento psíquico de militares frente al retiro laboral”, bajo la orientación del profesor Manuel Moreno.

En el nivel de maestría, el profesor Horacio Manrique, en el marco de la Maestría en Desarrollo Humano Organizacional de la Universidad EAFIT, ha dirigido los trabajos : "Gestión humana de orientación analítica: un camino para la responsabilización" (Pérez \& Lopera, 2016) y "Método clínico y trabajo analítico con grupos en el ámbito organizacional” (Ramírez, 2013); mientras que, en esta misma maestría, Lina María 
Gómez (2014), bajo la dirección de Isabel Lopera, desarrolló la tesis "Aplicaciones de la entrevista clínica en los procesos de selección de personal en la ciudad de Medellín”. A Sandra Moreno (2019), por su parte, le interesó caracterizar las experiencias de placer y sufrimiento en trabajadores de áreas de gestión humana comparando a profesionales de psicología con los de otras promociones.

En la Maestría en Psicología de la Universidad de San Buenaventura, Cali : Johnny Orejuela dirigió el trabajo titulado : "Experiencias de placer y sufrimiento en el trabajo de un grupo de altos ejecutivos de empresas privadas de la ciudad de Cali" (Londoño, 2016) ; en la Maestría en Psicología de la Universidad de Antioquia, la profesora Lina Marcela Gil y el mismo autor dirigieron la investigación: "Sentidos atribuidos a las experiencias de reconocimiento en el trabajo. Estudio de caso de investigadores de una universidad pública colombiana" (Gómez, 2018), la cual por su novedad, envergadura y rigor fue meritoria de una distinción. El profesor Julio Rubio, en la Maestría en Alta Dirección de Servicios Educativos de la Universidad de San Buenaventura, Cali : dirigió el trabajo : "Experiencias de malestar y sufrimiento en el trabajo de altos directivos de servicios educativos de instituciones públicas y privadas de la ciudad de Cali" (Rave, 2017).

41 En esta misma dirección de trabajos de grado de posgrado, la tesis doctoral del profesor Horacio Manrique, presentada en 2017, "Pensamiento intuitivo y deliberado en la experiencia de toma de decisiones intuitivas en directivos expertos colombianos", si bien no apela en su título a los significantes clínica del trabajo o de las organizaciones, sí analiza el fenómeno laboral de la toma de decisiones de directivos empresariales desde una perspectiva clínica. En síntesis, se encuentran cuatro tesis de doctorado desarrolladas por investigadores colombianos, relacionadas con el campo de las clínicas del trabajo y las organizaciones : la de Lina Marcela Gil, la de Carlos Mario Henao, presentada en 2016, la de Horacio Manrique y la de Sergio Oquendo (2019). No contamos aquí la tesis de Orejuela (2014b), "O mal-estar subjetivo derivado da fragmentação laboral", porque si bien fue desarrollada por un autor colombiano, se realizó en el marco del doctorado en psicología social del trabajo de la Universidad del Sao Paulo, bajo la dirección del profesor Sigmar Malvezzi.

Quizás la primera tesis doctoral desarrollada en el país, es decir con impronta específicamente colombiana, en la perspectiva propiamente dicha de la clínica del trabajo que se sirve directamente tanto de los trabajos de Christophe Dejours como de los planteamientos de Axel Honneth, fue "El reconocimiento en los discursos de evaluación de desempeño", desarrollada por el profesor Sergio Rene Oquendo Puerta, presentada en 2019, bajo la dirección del Doctor Rodrigo Muñoz, en el marco del doctorado en Administración de la Universidad de EAFIT.

Como consecuencia, en parte, y por la influencia de la presencia de profesor Héctor Bermúdez también formado en Sociología y Clínica del trabajo en Canadá, en la Maestría de Gestión Humana de la Universidad de Antioquia, comienzan a desarrollarse investigaciones en la perspectiva de la psicodinámica del trabajo a través de trabajos como "El placer y el displacer en el trabajo en un hospital público de Nechí", desarrollado por Alexandra Quinta y Diana Sanclemente (2019), bajo la orientación de los profesores Óscar Ortiz y Sergio Oquendo. Se encuentra también el trabajo "Placer y sufrimiento en auxiliares de ambulancia del Municipio de Medellín” de Juliana Muñoz Soto y Yoleni García Torres, presentada en 2019, bajo la dirección de Sergio Oquendo. 


\subsection{La principal producción escrita sobre las clínicas del trabajo en Colombia}

La producción escrita se encuentra dispersa en libros, capítulos de libros y artículos y ha venido en aumento en los últimos años. Se destacan el libro : Gramáticas actuales de la relación sujeto trabajo, en la que Sigmar Malvezzi et al. (2012) aborda la necesidad de una lectura clínica de la relación sujeto-trabajo en la era de las empresas red, así como los efectos subjetivos de malestar y sufrimiento en el trabajo derivados de la flexibilización laboral precarizante. En 2014, en el primer volumen del libro Psicología de las organizaciones y del trabajo: apuestas de investigación se publica por primera vez en un libro colombiano algo sobre las clínicas del trabajo, destacándose la colaboración de la profesora Ana Magnólia Mendes, quien junto con Luciane Araujo y Fernanda Duarte (Mendes et al., 2014), presenta su trabajo titulado Escucha política y clínica del sufrimiento en el trabajo: contribuciones desde las prácticas en clínica brasileña de psicodinámica del trabajo, y el trabajo del profesor Marcelo Ribeiro (2014), de la Universidad de São Paulo, titulado Problemas contemporáneos para a psicología do trabalho e das Organizações, en el cual toca explícitamente como un tópico relevante y actual el problema del sufrimiento en el trabajo.

En este mismo libro se publican por autores colombianos, apelando a los significantes clínica del trabajo y sufrimiento laboral por primera vez en la literatura académica nacional, los trabajos: "La identidad de la evaluación subjetiva del trabajo: de la satisfacción al sufrimiento laboral" (Orejuela, 2014c) y "Clínicas del trabajo : un estado de la cuestión" (Melo \& Orejuela, 2014). De cuño plenamente colombiano se destaca la publicación de los profesores Horacio Manrique y Carlos Mario Henao, en coautoría con Isabel Lopera, Juan Pérez y Victoria Ramírez (2015) quienes publican el que sería el primer libro en una perspectiva clínica y analítica de la relación sujeto-organización titulado: Clínica analítica de las organizaciones. Adicionalmente, y sin inscribirlo explícitamente en una clínica del trabajo, la profesora de la Universidad de Antioquia Lina Marcela Gil (2016) publica Psicología, Trabajo e Individuación, el cual se podría caracterizar como una lectura clínica del sujeto en el contexto laboral desde la perspectiva de la psicología de Gilbert Simondon. Cabe aclarar que estos dos últimos libros han sido publicados en coedición por el fondo editorial de la Universidad Eafit y la editorial San Pablo y son resultado de trabajos desarrollados por investigadores del Grupo de Investigación El método analítico y su relación con las ciencias sociales y humanas. En la misma línea clínica y simondoniana, el profesor Carlos Mario Henao (2019), de la Universidad EAFIT, publicó el libro Individuación y mediación analítica en contextos organizacionales.

Ampliando la difusión de la perspectiva clínica del trabajo en Colombia, en el segundo volumen del libro Psicología de las organizaciones y del trabajo : apuestas de investigación II se hicieron múltiples contribuciones. Allí, el profesor Thomas Périlleux (2016) de la Universidad Católica de Lovaina, presentó su trabajo titulado "Malestar y sufrimiento en el trabajo : una comprensión institucional". Por su parte, Armando García (2016), de la Universidad de Puerto Rico, presentó el capítulo "Experiencia clínica con un grupo de enfermeras en su lugar de trabajo, consideraciones teóricas y metodológicas". Las profesoras Lina Marcela Gil y Jennifer Ortiz (2016) presentaron el trabajo "Comunicación e individuación : las organizaciones en su dimensión transindividual", afianzando la perspectiva de Gilbert Simondon en el campo laboral. Por su parte, 
Victoria Ramírez y Horacio Manrique (2016) aportaron el trabajo "Método clínico y trabajo analítico con grupos en el ámbito organizacional".

En el mismo sentido, en el libro Abordajes psicoanalíticos a inquietudes sobre la subjetividad III, dirigido principalmente a un público psicoanalítico, se publicaron dos trabajos que buscan introducir las reflexiones desde las clínicas del trabajo en este campo, en el cual es escaso el conocimiento de esta perspectiva en la que se usa el arsenal conceptual heredado por Freud y Lacan, aplicado a la comprensión del sufrimiento en el contexto de trabajo con un enfoque clínico-crítico. Se trata de los capítulos: "Del malestar al sufrimiento : lo no dicho que se escucha a través del síntoma de un docente" (Murcia \& Orejuela, 2016), y también su contribución "Malestar, sufrimiento y síntoma: sus cualidades subjetivas y su comprensión en el mundo del trabajo".

En este esfuerzo de difusión editorial se suma el capítulo "El malestar laboral en las clases medias profesionales : el impacto de la fragmentación del trabajo" (Orejuela \& Patiño, 2016) en el libro Pensar las clases medias profesionales: trayectorias, paradojas, estrategias y malestares. Otros dos libros publicados en español por investigadores colombianos, haciendo uso del referencial psicoanalítico, que han contribuido al desarrollo de una perspectiva clínica del trabajo y las organizaciones son : Fantasías que crean empresa : elementos psicoanalíticos sobre la génesis de las empresas familiares (Ortiz \& Bermúdez, 2015); y El malestar en la empresa: un síntoma del sujeto contemporáneo (Villegas, 2015).

Quizás el primer libro en el país escrito en una perspectiva Clínica del Trabajo, alineado con la tradición psicodinámica de clínica brasilera y francesa es el libro Clínica del Trabajo : El Malestar subjetivo derivado de la fragmentación laboral (Orejuela, 2018), el cual aborda la discusión amplia y muy bien fundamentada sobre cómo las actuales condiciones de fragmentación y precarización del trabajo (menos salario, más exigencias, peores contratos, más barreras a la inserción laboral, más tiempo de trabajo intensificado y bajo presión y menos reconocimiento, etc.) producen efectos de malestar y sufrimiento subjetivo en el campo laboral. Este libro articula y cierra el hiato entre la psicología clínica y la psicología organizacional y del trabajo, para dar lugar a una comprensión clínica de la relación del sujeto con el trabajo, más allá de las preocupaciones eficientistas y pragmatistas que han dominado la reflexión sobre el campo de la salud mental en el trabajo en Colombia.

El libro Clínica del Trabajo de Orejuela Gómez tiene como principal mérito involucrar el lector en las cuestiones del trabajo y acompañarlo en la búsqueda de la profundización en su estudio. Su lectura enriquece la comprensión de la interfaz entre el trabajo y la existencia humana, aunque los interrogantes sigan renitentes, o incluso aumentados. Si la lectura de este libro multiplica las cuestiones en la mente del lector, esas nuevas inquietudes serán señales de su mayor dominio sobre ese objeto. (Malvezzi, 2019, p. 66).

51 Pasando a la producción de artículos publicados en español por autores colombianos se destacan a su vez: Gestión humana de orientación analítica: un camino para la responsabilización (Pérez \& Lopera, 2016); Una revisión crítica de la noción de sufrimiento presentada por la psicodinámica del trabajo (Orejuela \& Malvezzi, 2016) y Clínica analítica de las organizaciones : una propuesta conceptual (Manrique, Lopera, \& Pérez, 2018), estos dos últimos publicados en la Revista Trabalho (En)Cena.

Desde Montreal, Canadá, otro centro de producción del conocimiento sobre clínica del trabajo, el profesor colombiano Héctor Bermúdez, quien tuvo a su cargo la cátedra de Sociología de la Empresa y fue Investigador del Département de l'enseignement du 
management, de la Université HEC-Montréal, ha contribuido con la difusión de la perspectiva clínica del trabajo en español a través de trabajos como: Sobre la alienación subjetiva en la organización del trabajo actual : una observación participante en el comercio de la alimentación al detal (Bermúdez, 2016); Sociología Clínica y Psicodinámica del Trabajo en el estudio del placer y el sufrimiento en el trabajo cotidiano (Bermúdez, 2013); y apelando al referencial psicoanalítico para una lectura clínico social de la empresa publicó : ¿De la horda a la empresa? Una comparación preliminar de la empresa con la masa organizada, ya estudié antes por el autor.

Recientemente, María Alejandra Gómez y Paula Andrea Calderón (2017) publicaron un artículo en el que integran la categoría sufrimiento desde una perspectiva psicodinámica, comparando esta perspectiva con la de organización saludable, de cara a descifrar el fenómeno de la salud mental en el trabajo. Por su parte, Néstor PorrasVelásquez (2017) plantea un análisis de la relación entre la salud mental y el malestar humano en el trabajo.

Finalmente, vale la pena destacar que en el marco de la Conferencia Iberoamericana sobre Seguridad, Salud y Subjetividad en el Trabajo realizada en 2015, en la Universidad de San Buenaventura, Cali : se desarrolló el Panel Internacional sobre Clínicas del Trabajo, contando por primera vez en Colombia con la presencia de la profesora Ana Magnolia Mendes, de la Universidad de Brasilia, quien ofreció una conferencia sobre "La escucha clínica del sufrimiento en el trabajo : contribuciones de la clínica psicodinámica del trabajo brasilera". En este mismo panel, el autor principal del presente artículo participó con una ponencia titulada "El malestar subjetivo en el trabajo y sus condiciones de posibilidad : una lectura desde las clínicas del trabajo"; y la profesora Tania García Ramos, de la Universidad de Puerto Rico, cerró el panel con una ponencia sobre "La psicología y la salud ocupacional en Puerto Rico : complejizando la categoría de salud desde una mirada histórico-crítica".

Estas conferencias, que se encuentran en proceso con la editorial Bonaventuriana para la consolidación de un libro fueron organizadas por la Red Iberoamericana para la Promoción de la Seguridad Integral en el Trabajo (Ripsip), que en esta oportunidad fue liderada en Colombia por el profesor Octavio Alberto Orozco, director de la Especialización en Psicología de la Salud Ocupacional de la Universidad de San Buenaventura, Cali : posgrado que alojó y patrocinó el evento. Cabe aclarar que esta especialización es única en su tipo en Latinoamérica y es hasta el momento el primer posgrado en nuestro país dentro del cual se ofrecieron cursos electivos sobre la perspectiva clínica del trabajo y su relación con la salud ocupacional, y particularmente con la investigación y teorización sobre los riesgos psicosociales en el contexto laboral.

\subsection{Síntesis de la producción académica sobre la relación trabajo- subjetividad y clínica del trabajo en Colombia}

La tabla presentada en el Anexo, después de la bibliografía final, y que la completa para las referencias, sintetiza la producción académica directamente relacionada sobre clínica del trabajo, refiriéndose los trabajos de grados de los diferentes niveles de formación. 


\section{Conclusiones}

57 Se puede concluir que en Colombia las clínicas del trabajo y las organizaciones vienen desarrollándose con mayor fuerza en los últimos cinco años. Desde 2014, particularmente en Cali y Medellín, ha sido evidente su mayor difusión y alcance tanto en el número de publicaciones (artículos, capítulos y libros) como de presencia en la vida académica a través de la participación en congresos, paneles y actividades de formación (cursos, direcciones de tesis) a nivel de pregrado y posgrado; solo resta comenzar a hacer encuentros académicos especializados en esta área subdisciplinar: coloquios, simposios, congresos, etc.

La relación trabajo-subjetividad como foco de investigación tiene una relativa tradición en el campo de la Psicología Organizacional y del Trabajo en Colombia, no obstante, sólo en el último lustro ha comenzado a emerger como perspectiva especifica de análisis la Clínica del Trabajo en el país. La dilatación temporal de la llegada de la Clínica del Trabajo a Colombia ha tenido como principal obstáculo la limitada difusión de esta perspectiva en el idioma español, pues la mayoría de las producciones esta en francés y portugués. Aun así, la migración académica de algunos de los profesores de Psicología del Trabajo a Brasil facilitó por primera vez el contacto con esta perspectiva de análisis y campo subdisciplinar y transdisciplinar. Cabe destacar la presencia del profesor Sigmar Malvezzi de la Universidad de Sao Paulo como formador de varios de los doctores y magísteres colombianos en el campo POT, lo que se constituyó en una oportunidad, una interfaz, para conocer los trabajos de Christophe Dejours, Dominique Lhuilier y Ana Magnolia Méndes.

El contacto general con el contexto académico brasilero potencializó ampliamente las posibilidades de inserción de la perspectiva clínica del trabajo en el país, aún incipiente pero con amplias posibilidades de progreso apuntalados en los programas de formación de posgrado en universidades como EAFIT y la Universidad de Antioquia, en donde las formaciones de maestría, clases y trabajos de grado, incluyen de manera explícita y decidida la temática del placer y el sufrimiento en el trabajo y, en general, de investigación sobre los efectos del trabajo sobre la salud mental en las perspectivas de las Clínicas del Trabajo. Con seguridad la organización de eventos especializados en Clínica del Trabajo y la continuación de publicación de libros desde esta perspectiva serán en el futuro inmediato algunas de las formas como puede fomentar a institucionalizarse la clínica del trabajo en Colombia.

Por el momento, como aconteció en Brasil, los resultados de la investigación en Clínica del Trabajo están dispersos en formaciones de pregrado y posgrado, revistas de psicología, medicina, administración y salud ocupacional y congresos generales de psicología, que poco a poco fueron dando lugar a la aglutinación de la producción académica e investigativa en revistas y congresos especializados como lo son Trabalhio (En)cena y el Congreso Brasilero de Psicodinamica y Clínica del Trabajo, liderados por la profesora Ana Magnolia Mendes, de la Universidad de Brasilia, y Lilian Ghizoni, de la Universidad Federa de Tocantins, y demás miembros del Laboratorio de Psicodinámica y clínica del Trabajo de la Universidad de Brasilia, que se constituyen en un ejemplo a seguir en pos de la consolidación de una comunidad académica cuyo interés sea la investigación sobre la relación trabajo-subjetividad en una perspectiva especifica de la Clínica del Trabajo como campo sub y transdisciplinar. 
61 Cabe resaltar, una vez más, que, si bien los asuntos laborales han sido estudiados en Colombia y Latinoamérica desde hace algún tiempo, desde múltiples perspectivas y con diversos focos de análisis, en el que ha sido dominante, casi hegemónica, la perspectiva funcionalista-positivista muy frecuentemente alineada con la perspectiva capitalista (taylor-fordista) e instrumental del trabajo ; el tema de relación sujeto-trabajo en clave de sufrimiento y desde una perspectiva clínica es casi totalmente nuevo, como se ha querido mostrar con todo detalle aquí, compilando referencias útiles tanto para expertos como para quienes inician un acercamiento al campo en nuestro país. Aproximarse y difundir la perspectiva de las clínicas del trabajo, como un enfoque de conceptualización, investigación e intervención de la relación sujeto-trabajo, es más que necesario y pertinente ; es una perspectiva clínico-crítica que puede reintroducir un humanismo más que retórico en la gestión de los recursos humanos.

El desafío consiste, entonces, en difundir, generalizar y legitimar en mayor medida un uso más amplio de las aproximaciones clínicas en el contexto del trabajo, tanto en su dimensión aplicada (terapéutica) como investigativa (metodológica). Una clínica que supere la perspectiva "ortopédica psicologista" de referencia adaptacionista, que reduce la subjetividad a un conjunto de prescripciones consideradas para restaurar el bienestar en el trabajo, o para asegurar el adecuado desempeño en tareas de servicio. Se trata, por el contrario, de la clínica en una perspectiva alternativa que no se reduce a buscar el ajuste a las normas y a la adaptación a las limitaciones exteriores, sino que concibe el desarrollo de posibilidades individuales y colectivas, la exploración de las condiciones de trabajo, así como de sus procesos (Lhuilier, 2006). Una clínica que parte de comprender que el sufrimiento emerge de un desarrollo impedido, de una amputación del poder de actuar (Clot, 2009); que reconoce la dimensión subjetiva implicada en el trabajo humano y que concibe "la intervención como investigación acción a esta doble articulación : comprender para transformar y transformar para comprender" (Lhuilier, 2006. p. 19). He aquí otro desafío.

\section{BIBLIOGRAFÍA}

Andrade, V. (2014). Técnicas de análisis cualitativo en uso : exploración a través de la relación entre la identidad profesional y la agencia. In J. Orejuela (Ed.), Psicología de las Organizaciones y del trabajo: Apuestas de investigación (pp. 191-216). Cali : Bonaventuriana.

Andrade, V. (2015). Configuración de la identidad profesional desde la agencia como un recurso explicativo de la relación persona-trabajo (Tesis de doctorado). Instituto de Psicología, Universidad del Valle, Cali, Colombia.

Araujo, A. (2014). La gestión por resultado. Del capitalismo afectivo al acoso moral. In J. Orejuela, V. Andrade, \& M. Villamizar (Eds.), Psicología de las Organizaciones y del trabajo : Apuestas de investigación II (pp. 111-124). Cali : Bonaventuriana. 
Aristizabal, L. (2019). Vivencias de placer y sufrimiento en la formación de un grupo de doctorandos de la ciudad de Medellín (Tesis de Pregrado). Departamento de Psicología, Universidad Eafit, Medellín, Colombia.

Bendassolli, P. \& Soboll, L. (2011). Clínicas do trabalho : novas perspectivas para compreensão do trabalho na atualidade. São Paulo: Atlas.

Cardona, J. (2019). Placer y sufrimiento en trabajadores emprendedores (Tesis de Pregrado). Departamento de Psicología, Universidad Eafit, Medellín, Colombia.

Bermúdez, H. (2013). Sociología Clínica y Psicodinámica del Trabajo en el estudio del placer y el sufrimiento en el trabajo cotidiano. Revista Latinoamericana de Investigación en Organizaciones, Ambiente y Sociedad - Teuken Bidikay, 4(4), 153-180.

Bermúdez, H. (2016). Sobre la alienación subjetiva en la organización del trabajo actual : una observación participante en el comercio de la alimentación al detal. Contaduría y Administración, 62(1), 262-278. http://dx.doi.org/10.1016/j.cya.2016.10.004

Clot, Y. (2009). ¿El trabajo sin seres humanos? Psicología de los entornos de trabajo y de vida. Madrid : Modus Laborandi.

Correa, A. (2019). Vivencias de placer y malestar en pilotos de aerolíneas Charter colombianas (Tesis de Pregrado). Departamento de Psicología, Universidad Eafit, Medellín, Colombia.

Corredor, L., Páramo, A., \& Orejuela, J. (2018). Sentidos atribuidos al trabajo : un estudio sobre las generaciones ' $X$ ' y ' $Y$ ', en una empresa de servicios de Medellín. Revista Fórum Doctoral, 7, 1-38.

Dejours, C., \& Bègue, F. (2010). Trabajo y suicidio. Madrid : Modus Laborandi.

Del Carpio, S., Álvaro, J., \& Garrido, A. (2014). El significado del trabajo : aproximaciones teóricas y empíricas. In Orejuela, J. (Ed.), Psicología de las Organizaciones y del trabajo : Apuestas de investigación (pp. 131-159). Cali : Bonaventuriana.

Delgado, L. (2016). El contagio emocional en las organizaciones. Conceptos, hallazgos e implicaciones prácticas. In J. Orejuela, V. Andrade, \& M. Villamizar (Eds.), Psicología de las Organizaciones y del trabajo : Apuestas de investigación II (pp. 371-384). Cali : Bonaventuriana.

Díaz, F. (2017). Burnout, una metáfora para comprender las relaciones y contextos de trabajo desde la salud (Tesis de doctorado). Instituto de Psicología, Universidad del Valle, Cali, Colombia.

Fang, Y. (2009). La Fatiga laboral como una consecuencia en la salud derivada de la organización del trabajo. In E. Rentería \& M. Aguilar (Eds.), Psicología Organizacional y del Trabajo : Reflexiones y experiencias de investigación (pp. 363-390). Cali : Editorial Universidad del Valle.

Gálvez, L., \& Garcés, M. (2013). La identidad profesional y el desarrollo de carrera en mujeres gerentes. (Tesis de maestría). Instituto de Psicología. Universidad del Valle. Cali, Colombia.

García, F. (2014). Construcción de identidades profesionales de oficiales de la policía nacional de Colombia (Tesis de maestría). Instituto de Psicología, Universidad del Valle, Cali, Colombia.

García, A. (2016). Experiencia clínica con un grupo de enfermeras en su lugar de trabajo. Consideraciones teóricas y metodológicas. In J. Orejuela (Ed.), Psicología de las organizaciones y del trabajo : apuestas de investigación (pp. 459-470). Cali : Bonaventuriana.

García, Y. (2015). El sufrimiento del sujeto por el trabajo : una aproximación psicoanalítica. (Tesis de Pregrado). Facultad de Psicología, Universidad Católica de Pereira, Pereira, Colombia. 
García, M., \& Forero, C. (2014). La motivación y satisfacción laboral como determinantes asociados al cambio organizacional. In J. Orejuela (Ed.), Psicología de las Organizaciones y del trabajo : Apuestas de investigación (pp. 327-350). Cali : Bonaventuriana.

García, M., \& Vélez, H. (2016). Estrés y afrontamiento en el ámbito laboral. In J. Orejuela, V. Andrade, \& M. Villamizar (Eds.), Psicología de las Organizaciones y del trabajo : Apuestas de investigación II (pp. 489-515). Cali : Bonaventuriana.

Gil, L. (2016). Psicología, Trabajo e Individuación. Bogotá : San Pablo-Eafit.

Gil, L., \& Ortiz, J. (2016). Comunicación e individuación : las organizaciones en su dimensión transindividual, afianzando la perspectiva de Gilbert Simondon en el campo laboral. In J. Orejuela, V. Andrade, \& M. Villamizar (Eds.), Psicología de las Organizaciones y del trabajo : Apuestas de investigación II (pp. 303-326). Cali : Bonaventuriana.

Giraldo, A. (2015). Carrera, identidad y trayectorias en condiciones de trabajo fragmentado (Tesis de doctorado). Instituto de Psicología, Universidad del Valle, Cali, Colombia.

Giraldo, J., Aguilar, M., \& González, L. (2009). El Mobbing y la violencia en las organizaciones en Colombia. In E. Rentería, \& M. Aguilar (Eds.), Psicología Organizacional y del Trabajo : Reflexiones y experiencias de investigación (pp. 391-420). Cali : Editorial Universidad Del Valle.

Gómez, D. (2018). Sentidos atribuidos a las experiencias de reconocimiento en el trabajo. Estudio de caso de investigadores de una universidad pública colombiana (Tesis de Maestría). Maestría en Psicología, Universidad de Antioquia, Medellín, Colombia.

Gómez, I. (2009). Promoción de la salud en el lugar de trabajo. In E. Rentería, \& M. Aguilar (Eds.), Psicología Organizacional y del Trabajo : Reflexiones y experiencias de investigación (pp. 445-476). Cali : Editorial Universidad del Valle.

Gómez., L. (2014). Aplicaciones de la entrevista clínica en los procesos de selección de personal en la ciudad de Medellín (Tesis de Maestría). Maestría en Desarrollo Humano Organizacional, Universidad Eafit, Medellín, Colombia.

Gómez, M., \& Calderón, P. (2017). Salud mental en el trabajo : entre el sufrimiento en el trabajo y la organización saludable. Katharsis, 23, 177-201. https://doi.org/10.25057/issn.2500-5731

Grueso, M. (2016). Organizaciones saludables y procesos organizacionales e individuales : comprensión y retos. Bogotá : Universidad del Rosario.

Grueso, M., Antón, C., \& López-Santamaria, M. (2016). Actitudes frente a la vinculación laboral de personas en proceso de reintegración a la empresa colombiana : retos para la Psicología de las Organizaciones y del Trabajo. In J. Orejuela, V. Andrade, \& M. Villamizar (Eds.), Psicología de las Organizaciones y del trabajo : Apuestas de investigación II (pp. 167-194). Cali : Bonaventuriana.

Henao, C. (2019). Individuación y mediación analítica en contextos organizacionales. Medellín : Eafit. Lemos, M., Calle, G., Roldán, T., Valencia, M., Orejuela, J., \& Román-Calderón, J. (2019). Factores psicosociales asociados al estrés en profesores universitarios colombianos. Diversitas : Perspectivas en Psicología, 15(1), 59-70. https://doi.org/10.15332/s1794-9998.2019.0001.05

Lhuilier, D. (2006). Clinique du Travail. Paris : Érès.

Maca, D. (2019). El emprendimiento, gubernamentalidad y subjetividad : un abordaje de la política pública de emprendimiento en Colombia (Tesis de doctorado). Universidad del Valle, Cali, Colombia.

Malvezzi, S. (2019). The Clinics of Work : Subjective Distress Derived from Labour Fragmentation. International Journal of Psychological Research, 12(1), 66-67. https://doi.org/10.21500/20112084.4111 
Malvezzi, S., Orejuela, J., Chiuzi, R., Vesga, J., \& Riascos, W. (2012). Gramáticas actuales de la relación sujeto trabajo. Cali : Bonaventuriana.

Manrique, M. (2017). Pensamiento intuitivo y deliberado en la experiencia de toma de decisiones intuitivas en directivos expertos colombianos (Tesis de doctorado). Facultad de Psicología, Universidad del Norte, Barranquilla, Colombia.

Manrique, H., Henao, C. Lopera, I, Pérez, J., \& Ramírez, V. (2015). Clínica analítica de las organizaciones. Bogotá : San Pablo.

Manrique, H., Lopera, I., \& Pérez, J. (2018). Clínica analítica de las organizaciones : una propuesta conceptual. Revista Trabalho (En)Cena, 3(1), 23-34.

Melo, A., \& Orejuela, J. (2014). Clínicas del trabajo : un estado de la cuestión. In J. Orejuela (Ed.), Psicología de las Organizaciones y del trabajo : Apuestas de investigación (pp. 381-410). Cali : Bonaventuriana.

Mendes, A., Araujo, L, \& Duarte, F. (2014). Escucha política y clínica del sufrimiento en el trabajo : contribuciones desde las prácticas en clínica psicodinámica del trabajo brasilera. In J. Orejuela (Ed.), Psicología de las organizaciones y del trabajo : apuestas de investigación (pp. 25-46). Cali : Bonaventuriana.

Montoya, F., \& Panesso, A. (2014). Las estrategias de afrontamiento en una muestra de trabajadores con exposición a factores de riesgo psicosociales de una empresa manufacturera del norte del cauca. In J. Orejuela (Ed.), Psicología de las Organizaciones y del trabajo : Apuestas de investigación (pp. 467-494). Cali : Bonaventuriana.

Muñoz, J., \& García, Y. (2019). Placer y sufrimiento en auxiliares de ambulancia del Municipio de Medellín. (Tesis de Maestría). Maestría en Gestión Humana, Universidad de Antioquia, Medellín, Colombia.

Murcia, M., \& Orejuela, J. (2016). Del malestar al sufrimiento : lo no dicho que se escucha a través del síntoma de un docente. In M. Moreno, J. Orejuela, \& T. Calderón (Eds.), Abordajes psicoanalíticos a inquietudes sobre la subjetividad III (pp. 251-278). Cali : Bonaventuriana.

Neisa, C., Godoy, L., \& Tangarife, A. (2014). Variables moderadoras de la relación la fatiga laboral y accidentes de trabajo en personal operativo. In J. Orejuela (Ed.), Psicología de las Organizaciones y del trabajo : Apuestas de investigación (pp. 429-448). Cali : Bonaventuriana.

Oquendo, S. (2019). El reconocimiento en el discurso de la evaluación del desempeño : el caso de una gran empresa de Medellín (Tesis de Doctorado). Doctorado en Administración, Universidad Eafit, Medellín, Colombia.

Ordoñez, C., \& Mora, J. (2018). Experiencias de sufrimiento laboral en trabajadores (Tesis de Pregrado). Facultad de Psicología, Institución Universitaria Cesmag, Pasto, Colombia.

Orejuela, J. (2014a). Psicología de las Organizaciones y del trabajo : Apuestas de investigación. Cali : Bonaventuriana.

Orejuela, J. (2014b). o mal-estar subjetivo derivado da fragmentação laboral (Tesis de Doctorado). Doctorado en Psicologia Social del Trabajo, Instituto de Psicología, Universidad de São Paulo, São Paulo, Brasil.

Orejuela, J. (2014c). La identidad de la evaluación subjetiva del trabajo : de la satisfacción al sufrimiento laboral. In J. Orejuela (Ed.), Psicología de las organizaciones y del trabajo : apuestas de investigación. (pp. 163-190). Cali : Bonaventuriana. 
Orejuela, J. (2018). Clínica del trabajo : el malestar subjetivo derivado de la fragmentación laboral. Bogotá : San Pablo-Eafit.

Orejuela, J., Andrade, V., \& Villamizar, M. (2014). Psicología de las Organizaciones y del trabajo : Apuestas de investigación II. Cali : Bonaventuriana.

Orejuela, J., Bermúdez, R., Urrea, C., \& Delgado, L. (2013). Inserción laboral de jóvenes profesionales : el caso de los psicólogos bonaventurianos. Cali : Bonaventuriana.

Orejuela, J., \& Malvezzi, S. (2016). Una revisión crítica de la noción de sufrimiento presentada por la psicodinámica del trabajo. Trabalho (En)Cena, 1(1), 15-28.

Orejuela, J., \& Patiño, J. (2016). El malestar laboral en las clases medias profesionales : el impacto de la fragmentación del trabajo. In F. Sánchez, J. Orejuela, R. Bermúdez, V. Unas, \& J. Patiño (Eds.), Pensar las clases medias profesionales (pp. 61-86). Cali : Bonaventuriana.

Orejuela, J., \& Ramírez, A (2011). Aproximación cualitativa al estudio de la subjetividad laboral en profesionales colombianos. Pensamiento psicológico, 9(16), 125-144.

Ortiz, O., \& Bermúdez, H. (2015). Fantasías que crean empresa : elementos psicoanalíticos sobre la génesis de las empresas familiares. Bogotá : Ecoe y Unaula.

Peralta, C. (2009). La construcción de sujetos en las nuevas realidades laborales : reflexiones para su estudio desde la psicología organizacional y del trabajo. In E. Rentería, \& M. Aguilar (Eds.), Psicología Organizacional y del Trabajo : Reflexiones y experiencias de investigación : (pp. 53-76). Cali : Editorial Universidad Del Valle.

Pérez, J., \& Lopera, I. (2013). Gestión humana de orientación analítica : un camino para la responsabilización (Tesis de Maestría). Maestría en Desarrollo Humano Organizacional, Universidad Eafit, Medellín, Coombia.

Pérez, J., \& Lopera, I. (2016). Gestión humana de orientación analítica : un camino para la responsabilización. Revista de Administração de Empresas, 56(1), 101-113. https://doi.org/10.1590/ S0034-759020160109

Périlleux, T. (2016). Malestar y sufrimiento en el trabajo : una comprensión institucional. In J. Orejuela, V. Andrade, \& M. Villamizar (Eds.), Psicología de las organizaciones y del trabajo : apuestas de investigación II (pp. 101-110). Cali : Bonaventuriana.

Porras-Velásquez, N. (2017). Análisis de la relación entre la salud mental y el malestar humano en el trabajo. Equidad \& Desarrollo, 29, 161-178. http://dx.doi.org/10.19052/ed.4173

Pulido, H. (2009). Examinado las operaciones de la Psicología en su relación con el trabajo. In E. Rentería, \& M. Aguilar (Eds.), Psicología Organizacional y del Trabajo : Reflexiones y experiencias de investigación (pp. 99-112). Cali : Universidad del Valle.

Pulido, H. (2012). Reflexiones en torno al papel de la psicología en la constitución de la subjetividad en medio de las transformaciones de la organización del trabajo. Cuadernos De Psicología, 8(2), 69-84.

Pulido, H. (2013). ¿Y entonces ¿esto de la crítica que es ? De las relaciones de la psicología con el mundo del trabajo. Universitas Psychologica, 12(4), 1456-1467.

Pulido, H. (2018). Explorando la construcción de la subjetividad laboral : del ejecutor de oficios, al trabajador psicologizado. Revista de Psicología Universidad de Antioquia, 10(1), 237-258. https:// doi.org/10.17533/10.17533/udea.rp.v10n1a10.

Pulido, H., \& Carvajal, M. (2014). Pathologization and depathologization of the "free worker" in terms of the psychosocial risk. International Journal of Psychological Research, 7(2), 76-84. 
Quiroga, S., \& Valencia, A. (2016). La investigación como trabajo, ¿un escenario de placer, sufrimiento y lucha por el reconocimiento ?: un estudio en un grupo de ocho investigadores de la ciudad de Cali (Tesis de Pregrado). Facultad de Psicología, Universidad de San Buenaventura, Cali, Colombia.

Ramírez. V., \& Manrique, H. (2016). Método clínico y trabajo analítico con grupos en el ámbito organizacional. In J. Orejuela, V. Andrade, \& M. Villamizar (Eds.), Psicología de las Organizaciones y del trabajo : Apuestas de investigación II (pp. 415-436.). Cali : Bonaventuriana.

Rentería, E., \& Aguilar, M. (2009). Psicología Organizacional y del Trabajo : Reflexiones y experiencias de investigación. Cali : Universidad del Valle.

Ribeiro, M. (2014). Problemas contemporâneos para a psicología do trabalho e das organizações. In J. Orejuela (Ed.), Psicología de las Organizaciones y del trabajo : Apuestas de investigación (pp. 47-78). Cali : Bonaventuriana.

Romero, M. (2017). Significado del trabajo y sentido de la profesión académica : un estudio en el contexto colombiano (Tesis de doctorado). Instituto de Psicología, Universidad del Valle, Cali, Colombia.

Romero, M., Blanch, J., \& Rentería, E., (2016). Significado del trabajo y sentido de la profesión académica en el contexto flexible de la universidad colombiana. In M. Grueso (Ed.), Organizaciones saludables y procesos organizacionales e individuales : comprensión y retos (pp. 289-328). Bogotá : Universidad del Rosario.

Sanabria, S. (2016). El sufrimiento psíquico de militares frente al retiro laboral (Tesis de Especialización). Especialización en Psicología Clínica con orientación Psicoanalítica, Universidad de San Buenaventura, Cali, Colombia.

Sanín, A. (2016). Felicidad Laboral : Reflexiones sobre su aplicación al contexto laboral. Psicología del trabajo. Un entorno de factores psicosociales saludables para la productividad. México : Manual Moderno.

Torres, F. (2010). La identidad profesional de dirigentes de organizaciones no gubernamentales a partir de sus trayectorias (Tesis de maestría). Instituto de Psicología, Universidad del Valle, Cali, Colombia.

Uchida, S., Sznelwar, L., Barros, J., \& Lancman, S. (2011). O trabalhar em serviços de saúde mental : entre o sofrimento e a cooperação. Laboreal, 7(1), 28-41. http://laboreal.up.pt/revista/ artigo.php?id=48u56oTV65822353389453854:2

Vesga, J. (2009). El contrato psicológico y las nuevas realidades del trabajo. In E. Rentería, \& M. Aguilar (Eds.), Psicología Organizacional y del Trabajo : Reflexiones y experiencias de investigación (pp. 77-98). Cali : Editorial Universidad Del Valle.

Vesga, J. (2014). Evolución histórica del concepto “contrato psicológico. In J. Orejuela (Ed.), Psicología de las Organizaciones y del trabajo : Apuestas de investigación (pp. 243-250). Cali : Bonaventuriana.

Vesga, J. (2015). Contenido y extensión del contrato psicológico en trabajadores vinculados a una misma organización mediante distintas modalidades de trabajo (Tesis de doctorado). Universidad del Valle. Cali, Colombia.

Villegas, E. (2015). El malestar en la empresa : un síntoma del sujeto contemporáneo. Bogotá : San Pablo.

\section{ANEXOS}

Anexo 
Trabajos de grado según el nivel de formación, tipo de producto y relación directa con las Clínicas del trabajo

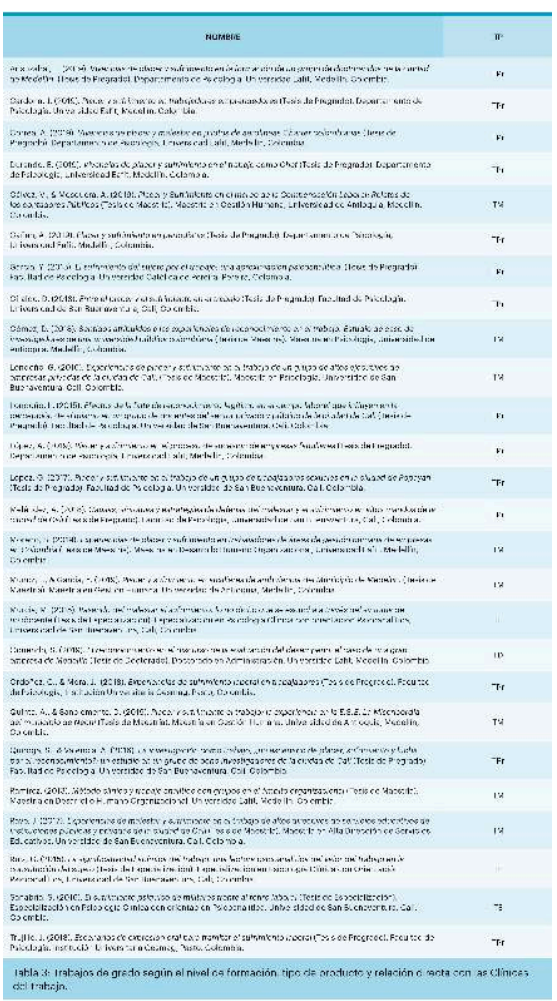

\section{RESÚMENES}

El propósito de este trabajo es presentar un balance sobre la investigación alrededor de las clínicas del trabajo en el contexto académico colombiano. El valor de este estado de la cuestión es que ofrece la posibilidad de representarnos y ubicar en el mapa académico una perspectiva alternativa de la psicología de la salud ocupacional. Para cumplir este cometido se propone discutir tres aspectos relevantes : primero, hacer una aproximación inicial a las condiciones que caracterizan las clínicas como un abordaje clínico-crítica de la relación trabajo-subjetividadsalud laboral; segundo, se indica como contexto predecesor : la investigación sobre la relación trabajo-subjetividad en Colombia, que en nuestro país ha sido generalmente abordado por el multicampo de la psicología del trabajo y las organizaciones. Posteriormente, se describe la progresiva emergencia e institucionalización de las clínicas del trabajo en Colombia (publicación, eventos, posgrados, tesis, etc.) en algunas universidades, principalmente de las ciudades de Cali y Medellín

O objetivo deste artigo é apresentar um balanço sobre a pesquisa em torno das clínicas do trabalho no contexto académico colombiano. $O$ interesse deste estado da arte é o de oferecer, simultaneamente, a possibilidade por si mesmo de uma representação, e uma perspectiva alternativa da psicologia da saúde ocupacional no mapa do mundo universitário. Para cumprir esse objetivo, propõe-se discutir três aspectos pertinentes. Primeiro, fazer uma abordagem inicial das condições que caracterizam as clínicas como abordagem clínico-crítica da relação trabalhosubjetividade-saúde. Em segundo lugar, a pesquisa sobre a relação trabalho-subjetividade é definida como um contexto predecessor. Na Colômbia, este tipo de pesquisa tem sido geralmente abordada pelo domínio interdisciplinar da psicologia do trabalho. Por fim, descreve-se a 
emergência e a institucionalização progressiva dos estudos que integram as clínicas do trabalho em algumas universidades na Colômbia (publicação, eventos, pós-graduação, teses, etc.), principalmente nas cidades de Cali e Medellín.

Cet article veut présenter un bilan des recherches menées en clinique du travail dans le contexte universitaire colombien. L'intérêt de cet état de l'art est de situer dans le champ du monde académique colombien la place qu'y prend une perspective de la psychologie de la santé au travail qui se veut alternative. L'article est organisé en trois volets. Le premier reprend les conditions qui sont propres à la clinique-critique de la relation travail-subjectivité-santé. Dans le second, nous définissons la recherche sur la relation travail-subjectivité comme préalable, car en Colombie ce type de recherche a généralement été intégré dans le large domaine de la psychologie du travail et des organisations. Enfin, nous décrivons l'émergence et l'institutionnalisation progressive des cliniques du travail dans quelques universités colombiennes, (publications, manifestations, études de troisième cycle, thèses, etc.), notamment dans les villes de Cali et Medellín.

The purpose of this document is to present an update on work clinics research in the Colombian academic context. The value of this state-of-the-art research is that it offers the possibility of representing ourselves and placing on the academic map an alternative perspective of the psychology of occupational health. Three relevant aspects will be discussed to fulfill this task. First, to make an initial approach to the conditions that characterize clinics as a clinical-critical approach to the work-subjectivity-occupational health relationship. Second, it is indicated as a predecessor context : research on the work-subjectivity relationship in Colombia, which in our country has been generally approached by the multi-field of work psychology and organizations. Finally, to describe the progressive emergence and institutionalization of work clinics in Colombia (publication, events, postgraduate studies, thesis, etc.) in some universities, mainly in the cities of Cali and Medellin.

\section{ÍNDICE}

Palavras-chave: trabalho, subjetividade, clínicas do trabalho, prazer e sofrimento Palabras claves: trabajo, subjetividad, clínicas del trabajo, placer y sufrimiento Mots-clés: travail, subjectivité, cliniques du travail, plaisir et souffrance Keywords: work, subjectivity, clinics of work, pleasure and suffering

\section{AUTORES}

\section{JOHNNY OREJUELA}

Departamento de Psicología, Universidad Eafit, Calle 27 D sur 28-50, Medellín, Antioquia, Colombia jorejue2@eafit.edu.co

\section{MARÍA DEL MAR PÉREZ}

Universidad Antonio Nariño ; Calle $5 \mathrm{~b} 4 \mathrm{~N}^{\circ} 36$ b 40, Cali : Valle, Colombia

mariadelmarpereza@hotmail.com 


\section{ANDRÉS VÁSQUEZ}

Departamento de Psicología, Universidad Eafit ; Calle 3 sur 37 A 25, Medellín, Antioquia, Colombia avasqueo@eafit.edu.co 